\title{
A TRANSFORMATION THEOREM ON SPECTRAL MEASURES ${ }^{1}$
}

\section{HABIB SALEHI}

1. Introduction. Let $\Theta$ be a $\sigma$-algebra of subsets of a given space $\Omega$, and let $E$ be a countably additive projection-valued measure for a Hilbert space $\mathfrak{H C}$, i.e., for each $B \in \Theta, E(B)$ is a projection operator on F such that $E\left(\cup_{k} B_{k}\right)=\sum_{k} E\left(B_{k}\right)$ whenever $\left\{B_{k}\right\}$ is a sequence of disjoint sets in $B$ and $E(\Omega)=I$, where $I$ denotes the identity operator on $\mathfrak{H}$. We will refer to $(\Omega, \mathbb{B}, \mathfrak{H}, E)$ as a spectral measure for the Hilbert space $\mathfrak{H}$.

For each element $x$ in $\mathfrak{F}$ the function $|E(\cdot) x|^{2}$ is a countably additive nonnegative measure on $B$ such that $|E(\Omega) x|^{2}=|x|^{2}<\infty$. If $\phi$ is a $B$-measurable function defined on $\Omega$, then the integral $\int_{\Omega} \phi(\omega) E(d \omega)$ is a well-defined linear operator $T$ (not necessarily bounded) in $\mathcal{H}$ with domain

$$
D_{T}=\left\{x: x \in \operatorname{HC} \& \int_{\Omega}|\phi(\omega)|^{2}|E(d \omega) x|^{2}<\infty\right\} .
$$

It is a simple result that if $x \in D_{T}$, then

$$
|T x|^{2}=\int_{\Omega}|\phi(\omega)|^{2}|E(d \omega) x|^{2} .
$$

In this paper we propose to establish the following theorem concerning transformation of spectral measures for a Hilbert space $\mathfrak{H}$.

2. Theorem. Let

(i) $\left(\Omega_{1}, \Theta_{1}, \mathfrak{H}, E_{1}\right)$ be a spectral measure for a Hilbert space $\mathfrak{H C}$,

(ii) $T$ be a single-valued transformation on $\Omega_{1}$ into a space $\Omega_{2}$,

(iii) $\beta_{2}=\left\{B: B \subseteq \Omega_{2}\right.$ and $\left.T^{-1}(B) \in \Theta_{1}\right\}$,

(iv) for each $B \in Q_{2}$,

$$
E_{2}(B)=E_{1}\left(T^{-1}(B)\right) .
$$

Then (a) $\left(\Omega_{2}, \Theta_{2}, \mathcal{F}, E_{2}\right)$ is a spectral measure for the Hilbert space $\mathfrak{F}$. (b) For each $\mathrm{B}_{2}$-measurable function $\phi$ on $\Omega_{2}$ we have

$$
\int_{\Omega_{1}} \phi\left(T\left(\omega_{1}\right)\right) E_{1}\left(d \omega_{1}\right)=\int_{\Omega_{2}} \phi\left(\omega_{2}\right) E_{2}\left(d \omega_{2}\right)
$$

Received by the editors March 14, 1966.

1 This research was partially supported by Public Health Service Research Grant No. NIH-GM-13138-01 from the National Institute of General Medical Sciences. 
Several times in the proof we will make use of a lemma on transformation of scalar-valued measures. Because of great similarity between this lemma and our theorem and for ease of reference, the lemma is stated below. (cf. [3, Theorem C, p. 163]).

3. Lemma. If $T$ is a measurable transformation from a measure space $(X, \mathcal{S}, \mu)$ into a measurable space $(\boldsymbol{Y}, \mathrm{J})$, and if $\mathrm{g}$ is an extended real-valued measurable function on $Y$, then

$$
\int_{Y} g d\left(\mu T^{-1}\right)=\int_{X}(g T) d \mu
$$

in the sense that if either integral exists, then so does the other and the two are equal.

Proof of Theorem 2. (a) It is obvious that for each $B \in B_{2}$, $E_{2}(B)$ is a projection operator on $\mathcal{H C}$ and $E_{2}\left(\Omega_{2}\right)=I$. If $\left\{B_{k}\right\}$ is a disjoint sequence of sets in $B_{2}$, then $\left\{T^{-1}\left(B_{k}\right)\right\}$ is a sequence of disjoint sets in $B_{1}$, and hence

$$
\begin{aligned}
E_{2}\left(\bigcup_{k} B_{k}\right) & =E_{1}\left(T^{-1}\left(\bigcup_{k}^{\bigcup} B_{k}\right)\right)=E_{1}\left(\bigcup_{k} T^{-1}\left(B_{k}\right)\right) \\
& =\sum_{k} E_{1}\left(T^{-1}\left(B_{k}\right)\right)=\sum_{k} E_{2}\left(B_{k}\right) .
\end{aligned}
$$

(b) We give the proof of (b) in two steps.

(Step 1). For a simple function $\phi=\sum_{k=1}^{n} a_{k} \chi_{B_{k}}$, where $\chi_{B_{k}}$ is the indicator function of $B_{k} \in \mathbb{B}_{2}$,

$$
\begin{aligned}
\int_{\Omega_{2}} \phi\left(\omega_{2}\right) E_{2}\left(d \omega_{2}\right) & =\int_{\Omega_{2}}\left(\sum_{k=1}^{n} a_{k} \chi_{B_{k}}\right)\left(\omega_{2}\right) E_{2}\left(d \omega_{2}\right)=\sum_{k=1}^{n} a_{k} E_{2}\left(B_{k}\right) \\
& =\sum_{k=1}^{n} a_{k} E_{1}\left(T^{-1}\left(B_{k}\right)\right) \\
& =\int_{\Omega_{1}}\left(\sum_{k=1}^{n} a_{k} \chi_{T^{-1}\left(B_{k}\right)}\right)\left(\omega_{1}\right) E_{1}\left(d \omega_{1}\right) \\
& =\int_{\Omega_{1}} \phi\left(T\left(\omega_{1}\right)\right) E_{1}\left(d \omega_{1}\right) .
\end{aligned}
$$

(Step 2). Let $\phi$ be any $B_{2}$-measurable function on $\Omega_{2}$, and let

$$
T_{1}=\int_{\Omega_{1}} \phi\left(T\left(\omega_{1}\right)\right) E_{1}\left(d \omega_{1}\right) \& T_{2}=\int_{\Omega_{2}} \phi\left(\omega_{2}\right) E_{2}\left(d \omega_{2}\right) .
$$


We first show that $T_{1}$ and $T_{2}$ have the same domain, i.e., $D_{T_{1}}=D_{T_{2}}$. For each $x$ in $\mathcal{H}$, by Lemma 3 ,

$$
\int_{\Omega_{1}}\left|\phi\left(T\left(\omega_{1}\right)\right)\right|^{2}\left|E_{1}\left(d \omega_{1}\right) x\right|^{2}=\int_{\Omega_{2}}\left|\phi\left(\omega_{2}\right)\right|^{2}\left|E_{2}\left(d \omega_{2}\right) x\right|^{2} .
$$

Now

$$
\begin{aligned}
x \in D T_{2} & \Leftrightarrow \int_{\Omega_{2}}\left|\phi\left(\omega_{2}\right)\right|^{2}\left|E_{2}\left(d \omega_{2}\right) x\right|^{2}<\infty \quad \text { by (1) } \\
& \Leftrightarrow \int_{\Omega_{1}}\left|\phi\left(T\left(\omega_{1}\right)\right)\right|^{2}\left|E_{1}\left(d \omega_{1}\right) x\right|^{2}<\infty \quad \text { by }(2) \\
& \Leftrightarrow x \in D T_{1} \quad \text { by }(1) .
\end{aligned}
$$

Hence $D_{T_{1}}=D_{T_{2}}$.

If $x \in D_{T_{2}}$, then by (1) and (3), $\int_{\Omega_{2}}\left|\phi\left(\omega_{2}\right)\right|^{2}\left|E_{2}\left(d \omega_{2}\right) x\right|^{2}$ is equal to $\int_{\Omega_{1}}\left|\phi\left(T\left(\omega_{1}\right)\right)\right|^{2}\left|E_{1}\left(d \omega_{1}\right) x\right|^{2}<\infty$. Therefore there exists a sequence of simple functions $\left\{\phi_{n}\left(\omega_{2}\right)\right\}_{n=1}^{\infty}$ on $\Omega_{2}$ such that

$$
\lim _{n \rightarrow \infty} \int_{\Omega_{2}}\left|\phi_{n}\left(\omega_{2}\right)-\phi\left(\omega_{2}\right)\right|^{2}\left|E_{2}\left(d \omega_{2}\right) x\right|^{2}=0 .
$$

It easily follows, by (3) and (4), that the sequence of simple functions $\left\{\phi_{n}\left(T\left(\omega_{1}\right)\right)\right\}_{n=1}^{\infty}$ on $\Omega_{1}$ satisfies the relation

$$
\lim _{n \rightarrow \infty} \int_{\Omega_{1}}\left|\phi_{n}\left(T\left(\omega_{1}\right)\right)-\phi\left(T\left(\omega_{1}\right)\right)\right|^{2}\left|E_{1}\left(d \omega_{1}\right) x\right|^{2}=0 .
$$

From (2), (4) and (5) it follows that

(6)

$$
\begin{gathered}
\left\{\int_{\Omega_{2}} \phi_{n}\left(\omega_{2}\right) E_{2}\left(d \omega_{2}\right)\right\} x \rightarrow T_{2} x, \quad \text { as } n \rightarrow \infty, \\
\left\{\int_{\Omega_{1}} \phi_{n}\left(T\left(\omega_{1}\right)\right) E\left(d \omega_{1}\right)\right\} x \rightarrow T_{1} x, \text { as } n \rightarrow \infty .
\end{gathered}
$$

Now

$$
\begin{aligned}
T_{2} x & =\lim _{n \rightarrow \infty}\left\{\int_{\Omega_{2}} \phi_{n}\left(\omega_{2}\right) E_{2}\left(d \omega_{2}\right)\right\} x \quad \text { by }(6) \\
& =\lim _{n \rightarrow \infty}\left\{\int_{\Omega 1} \phi_{n}\left(T\left(\omega_{1}\right)\right) E_{1}\left(d \omega_{1}\right)\right\} x \quad \text { by (Step 1) } \\
& =T_{1} x \quad \text { by }(6) .
\end{aligned}
$$

Hence $T_{1}=T_{2}$. Q.E.D. 


\section{REFERENCES}

1. N. Dunford and J. Schwartz, Linear operators, Part II: Spectral theory, Interscience, New York, 1963.

2. P. R. Halmos, Introduction to Hilbert space, Chelsea, New York, 1957.

3. - Measure theory, Van Nostrand, New York, 1961.

4. F. J. Murray, An introduction to linear transformations in Hilbert space, Princeton Univ. Press, Princeton, N. J., 1941.

Michigan State University

\section{THE MOMENTS OF RECURRENCE TIME}

\section{J. WOLFOWITZ ${ }^{1}$}

In connection with Poincaré's recurrence theorem Kac [1] obtained the mean of the recurrence time (formula (3) below) and the author [2] gave a very simple proof of this result. Recently Blum and Rosenblatt [3] obtained ${ }^{2}$ the higher moments (formula (2) below). In the present note we obtain both results by an exceedingly simple and perspicuous argument. This note is entirely self-contained.

Let $\Omega$ be a point set, $m$ a probability measure on $\Omega$, and $T$ a one-toone ergodic measure-preserving transformation of $\Omega$ into itself. Let $A \subset \Omega$ be such that $m(A)>0$. For any point $a$ in $\Omega$ let $n(a)$ be the smallest positive integer such that $T^{n} a \in A$; if no such integer exists let $n(a)=\infty$. Define $A_{k}=\{a \in A \mid n(a)=k\}, \bar{A}=\Omega-A$, and $\Gamma_{k}$ $=\{a \in \bar{A} \mid n(a)=k\}$. Borrowing the notation of [3] we will define

$$
p_{n}=m\left\{\Gamma_{n} \cup \Gamma_{n+1} \cup \ldots\right\},
$$

for $n \geqq 1$. We will also make use of the usual combinatorial symbol $(k)_{j}=k(k-1) \cdots(k-j+1)$ for $k$ and $j$ posilive integers, with $(k)_{0}=1$.

Our object will be to prove that

$$
D_{j}=\int_{A}[n(a)]_{j} d m=j(j-1) \sum_{k=j-2}^{\infty}(k)_{(j-2)} p_{k+1}
$$

Received by the editors August 1, 1966.

1 Fellow of the John Simon Guggenheim Memorial Foundation. Research supported in part by the U. S. Air Force under Contract AF 18(600)-685 with Cornell University.

2 These moments have also been obtained by F. H. Simons, Notice \#40 of the Eindhoven Technical School, December 23, 1966. 